\title{
What Happens When Proton Meets Randomization: Is There a Future for Proton Therapy?
}

\author{
Feng-Ming (Spring) Kong, Indiana University School of Medicine, Indianapolis, IN
}

See accompanying article on page 1813

The use of proton therapy has been a topic of debate for years. In the article that accompanies this editorial, Liao and colleagues ${ }^{1}$ report the first randomized study to assess the value of proton therapy compared with photon intensity-modulated radiotherapy (IMRT) in non-small-cell lung cancer (NSCLC). Completion of this study is not trivial because the evaluation of the benefit of a new technology rarely has been done during the century-long history of radiation oncology practice. A trial on the effectiveness of proton technology is particularly timely with the growing number of proton facilities in the United States and worldwide and its implication for value-based medicine.

Is a randomized trial needed for an advanced radiation technology with a clear benefit in terms of radiation dosimetry, such as proton therapy? ${ }^{2-4} \mathrm{~A}$ consensus has not been reached in the field with regard to this question. Although some ask for clinical outcome data, ${ }^{2}$ some may believe that a randomized trial is not needed because the dose to the tumor target can be increased, which means that higher tumor control probabilities and/or the dose to normal, nontumor-containing volumes can be decreased, thereby reducing the risks of normal tissue complications. ${ }^{3}$ Some may even argue that conducting a trial to test the significance of such a treatment is unethical, ${ }^{4}$ like performing a randomized study to test the value of parachutes, because it will put patients at risk for unneeded radiation complications. Such beliefs have been reflected in the history of radiotherapy technology advancement. From the first uses of $\mathrm{x}$-rays and radium for cancer treatment in the early 1900s, to kilovoltage (superficial) $\mathrm{x}$-ray machines and the era of cobalt-60 and megavoltage two-dimensional treatment, to Linacbased three-dimensional conformal technology and the current widespread use of IMRT, technologies have been developed and implemented routinely in the clinic without randomized trials. Similar scientific and ethical arguments were made erroneously for surgical and medical oncology disciplines, such as for breast cancer, until randomized trials subsequently showed that radical mastectomy was not better than breast-conserving therapy and that high-dose chemotherapy with stem-cell rescue was not beneficial in metastatic breast cancer. ${ }^{5,6}$

Protons have been recognized for their physical dosimetric advantage in phantom and model studies as a result of the unique dose distribution described by the Bragg peak (sparing normal tissue distal to the target) and branded as "the sharpest scalpel for cancer treatment" shown in one advertisement. The concept of using protons for cancer therapy was first developed by Robert Wilson, $\mathrm{PhD}$, early in
$1946^{7}$; the first patient was treated in 1954 in the Berkeley Radiation Laboratory; and the first fractionated treatment was performed in $1974 .^{8}$ However, implementation in clinical practice has been slow mainly because of the high cost of building the machine and the corresponding facility (a multiroom proton center costs approximately 40 times more than a conventional megavoltage photon radiation or IMRT facility ${ }^{9}$ ) as well as challenges in developing and implementing reliable dose-computation approaches. In addition, proton treatment and machine maintenance are much more expensive than photon therapy. Although 9,116 patients were treated with protons over 41 years at a joint program of Harvard Cyclotron Laboratory and the Massachusetts General Hospital before the cyclotron was shut down in 2002, hospital-based proton machines were not built until 1989 in the United Kingdom and 1990 in Loma Linda, California. Initially, protons were used mainly on fixed tumors, such as those in the base of the skull, and in pediatric patients. Could lung cancer, which presents a moving target with uncertainty of proton attenuation in low-density lung tissues, be treated effectively and cost-effectively? Comparative clinical outcome data are needed for patients and their families to choose a cancer treatment modality that is not readily available, for physicians to make treatment recommendations, for investors/industry to determine where to spend resources, for insurance companies and government to make reimbursement policies, and for researchers to know how and where to focus their efforts. Thus, a randomized trial is needed to generate unbiased evidence for this extremely costly technology.

The randomized trial reported by Liao and colleagues ${ }^{1}$ aimed to determine whether patients treated with proton therapy would have a lower risk of grade $\geq 3$ radiation pneumonitis (RP) in locally advanced NSCLC. The study hypothesized a $10 \%$ reduction in grade $\geq 3 \mathrm{RP}$ for the passive scattering proton therapy (PSPT) arm compared with the photon IMRT arm without compromise of local tumor control. No attempt was made in this study to improve tumor control; the rationale was only to decrease toxicity.

In contrast to the largest retrospective study of patients from the National Cancer Database, ${ }^{10}$ this prospective randomized study failed to prove superiority of proton therapy. Instead, the PSPT arm had $10.5 \%$ grade $\geq 3 \mathrm{RP}$ compared with only $6.5 \%$ in the IMRT arm, despite a significant reduction in low-dose volume in the dosimetric histograms for the PSPT arm. Significant dosimetric sparing of the heart and esophagus in the proton arm was found. The primary study outcomes of grade $\geq 3 \mathrm{RP}$ and local failure were comparable with 


\begin{tabular}{|c|c|c|c|}
\hline Study & Radiation Technology & $\begin{array}{c}\text { Grade } \geq 3 \\
\text { Radiation } \\
\text { Pneumonitis, } \\
\%\end{array}$ & $\begin{array}{c}\text { 2-Year } \\
\text { Tumor } \\
\text { Control, } \\
\%\end{array}$ \\
\hline \multirow{2}{*}{ Liao et $\mathrm{al}^{1}$} & PSPT arm: 3D, 66 or $74 \mathrm{~Gy}$ & 11 & $65-66+$ \\
\hline & IMRT arm: 66 Gy or $74 \mathrm{~Gy}$ & 7 & $69-70 \dagger$ \\
\hline \multirow[t]{2}{*}{ RTOG $617^{11}$} & 74-Gy arm: 3D-CRT/IMRT & 7 & 61 \\
\hline & 60-Gy arm: 3D-CRT/IMRT & 4 & 69 \\
\hline \multirow[t]{2}{*}{ PROCLAIM ${ }^{12}$} & $\begin{array}{l}\text { Pemetrexed + cisplatin } \\
\text { arm: } R^{*} 60-66 \mathrm{~Gy}\end{array}$ & $<3$ & 63 \\
\hline & 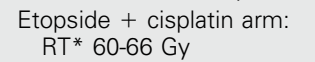 & $<3$ & 54 \\
\hline UMCC2007123 & $\begin{array}{l}\text { PET-guided ART; 3D-CRT; } \\
\text { median dose, } 83 \mathrm{~Gy}\end{array}$ & 7 & 82 \\
\hline \multicolumn{4}{|c|}{$\begin{array}{l}\text { Abbreviations: 3D, three-dimensional; 3D-CRT, three-dimensional conformal } \\
\text { radiation therapy; ART, adaptive radiation therapy; IMRT, intensity-modulated } \\
\text { radiation therapy; PET, positron emission tomography; PSPT, passive scattering } \\
\text { proton therapy; RT, radiation therapy. } \\
{ }^{*} \text { RT technology not specified. } \\
\text { tNumbers approximated visually from Fig. } 3 \mathrm{C}\end{array}$} \\
\hline
\end{tabular}

reports from recent studies such as the RTOG 0617, ${ }^{11}$ PROCLAIM, ${ }^{12}$ and UMCC2007123 $3^{13}$ for locally advanced NSCLC treated with concurrent chemoradiation (Table 1). The median survival of 26.1 months for PSPT and 29.5 months after IMRT were comparable with that of a similar patient population in RTOG 617 treated with standard conformal photon therapy. ${ }^{12}$

What are the potential reasons that this study failed to show decreased toxicity of proton therapy in locally advanced NSCLC? First, from a physical dosimetry aspect, this trial demonstrated that the PSPT arm had only significantly better dosimetry for lung volumes exposed to lower doses ( 5 to $10 \mathrm{~Gy}$ ) but no significant changes in mean lung dose and lung volumes at $\geq 20 \mathrm{~Gy}$, which means that PSPT had only a limited or no advantage over IMRT, even in physical lung dosimetry, and that this may partially explain the negative clinical results.

Second, the maturity of the proton and photon radiation planning could explain the insignificant difference in lung dosimetry. Practice makes perfect, and a learning curve is a reality for any new technology. Photon-based IMRT is in its prime, whereas proton therapy was new to the team at the time of the study and limited by the number of beams and beam angles. This claim is supported by evidence that more patients had superior PSPT plans at a later period in the trial and all RP events occurred during the earlier period of trial enrollment for the PSPT arm but were spread out evenly throughout the trial for IMRT. However, the trial demonstrated significant learning for both arms, and smaller tumors also likely contributed to less grade $\geq 3 \mathrm{RP}$ in the later period of the trials.

Third, the biologic effect of proton therapy may differ from what was modeled. The investigators seemed to intend to decrease lung volumes of lower doses according to the data from their historical photon studies. Even for photon therapy, how different dose volumes, such as a little dose to a lot of lung or a lot of dose to a little lung, affect the risk of lung damage is unclear. Even after photon therapy, although lung volume at 5 Gy was reportedly a significant factor for RP in some studies, more studies reported mean lung dose and volume receipt of $>20 \mathrm{~Gy}$ as significant factors. ${ }^{14}$ Proton therapy contributes several additional layers of uncertainty to the dose computation model. The biologic effect of proton therapy differs from that of photon therapy (although probably not by a lot). For example, the proton dose in the shoulder region differs from that in the Bragg peak, and the same proton dose may have different effects from photons for tumor and lung tissue.

Fourth, some imbalance in specific details between the PSPT and IMRT arms may have existed. For example, the margins for planning target volume were uniformly defined for the IMRT arm, but for proton planning, each beam had an individual and unique planning target volume expansion from the internal target volume, which also considered the range beam uncertainty. The use of adaptive planning may not have been used similarly between the two arms: the study allowed computed tomography scan and, as indicated, adaptive replanning at 2, 3, 4, and 7 weeks. Frequent adaptive radiotherapy planning might be more logistically challenging for the PSPT arm. Whether a difference existed in frequency of adaptive plan between the arms is unclear. More importantly, the effects of setup uncertainty and patient respiratory motion could have had a greater effect on the PSPT arm.

Finally, the study design in terms of end point definition, control of confounding factors, and dealing with the lung dosimetric restriction may have confounded the results. The end point of $\mathrm{RP}$ was defined as in-field radiographic changes, which may have excluded some patients with more serious RP with diffuse and extensive changes outside the radiotherapy fields. The confounding for the high-risk factors for RP, such as concurrent carboplatin and paclitaxel $^{15}$ and use of adjuvant taxotere, ${ }^{16}$ were not controlled. A significant number of patient denials for IMRT and insurance denials for PSPT after random assignment could also have resulted in a biased comparison between arms (which would not necessarily have been in favor of protons). More importantly, the study required patients to meet dosimetric limits for both the PSPT and the IMRT arms, which may have resulted in not being able to enroll patients who would most likely benefit from protons.

In summary, this randomized trial showed no benefit of proton therapy to reduce serious lung toxicity in the treatment of locally advanced NSCLC compared with IMRT with the technology available at that time. This finding challenges the assumption on the part of many that protons would certainly be superior and emphasizes the importance of evidence-based medicine and randomized trials. The debate will continue, and patients and physicians will continue to be challenged with the decision of whether to use proton therapy in lung cancer. Whether a better planning technique such as proton intensity modulation or pencil beam scanning would have generated different results is hard to predict. Personally, as a radiation oncologist, I would not recommend proton therapy for NSCLC outside a clinical trial setting until a clinical benefit is demonstrated in a prospective randomized study.

Is there a future for proton therapy? The results from Liao and colleagues ${ }^{1}$ suggest a dismal future in locally advanced NSCLC because the PSPT arm with significantly less lung volume receiving lower doses as well as significantly better dosimetry to the heart and esophagus did not lead to less lung toxicity or better survival (numerically higher rates of lung toxicity and shorter median survival instead). Although negative results from a phase II study in NSCLC cannot exclude the potential benefit of proton therapy in other clinical situations, such as for pediatric patients, and the cost of proton therapy will be significantly reduced by newer technological changes, this trial should at least cause some pause in hospitals that are building these facilities for 
competitive reasons and not for cost-effectiveness reasons. We should also learn from the experience of the Scripps Proton Therapy Center in San Diego, California, a center that cost \$165 million that started operations in 2011 and then filed for bankruptcy on March 1, 2017. Despite the rapid expansion of proton centers in the United States and in China, additional research clearly needs to speed up the learning curve for proton therapy, to study its biologic effects for both tumor and normal tissue, to determine the dosimetric effects on the clinical outcome, and to capture accurately the impact of setup/organ motion effects on the dose actually given to patients. A phase III study, RTOG 1308 (ClinicalTrials.gov identifier: NCT01993810) is ongoing for stage III NSCLC, but unfortunately, it is facing the challenge of slow accrual.

On the basis of the Liao et $\mathrm{al}^{1}$ findings, however, one may also look to the future of proton therapy optimistically. With the availability of more gantry angles, better imaging guidance, more-accurate dose computation for moving lung cancer targets and low-density of lung tissue, and more-advanced treatment planning technology like pencil beam scanning, we can generate remarkably better plans at every tumor dose level that lead to meaningful benefits for many patients in the clinic and are proven as cost-effective treatment in some specific disease settings. ${ }^{17}$ For future randomized trials, insurance approval of proton therapy and demonstration of a clear and substantial benefit of proton therapy in treatment plans for the same patient are important. The randomized trial should only include patients for whom the use of protons provides a better dosimetric plan. Such a randomized trial will identify patients with proven dosimetric superiority from proton planning to demonstrate whether such a dosimetric advantage can be translated into clinical benefit. Another possible advantage of protons to investigate is that by delivering less dose to much of the body (if achievable), protons may decrease radiation-mediated immune suppression ${ }^{18}$ and thereby improve survival in patients with NSCLC. With decreased costs, improved delivery systems, decreased doses to normal tissues including immune system, and improved understanding of the biology, proton therapy may yet be proven to be a cost-effective cancer treatment of specific diseases in specific settings.

\section{AUTHOR'S DISCLOSURES OF POTENTIAL CONFLICTS OF INTEREST}

Disclosures provided by the authors are available with this article at jco.org.

\section{REFERENCES}

1. Liao Z, Lee JJ, Komaki R, et al: Bayesian adaptive randomization trial of passive scattering proton therapy and intensity-modulated photon radiotherapy for locally advanced non-small-cell lung cancer. J Clin Oncol 10.1200/JCO.2017.74.0720
2. Tepper JE: Protons and parachutes. J Clin Oncol 26:2436-2437, 2008

3. Glimelius B, Montelius A: Proton beam therapy - do we need the randomised trials and can we do them? Radiother Oncol 83:105-109, 2007

4. Goitein M, Cox JD: Should randomized clinical trials be required for proton radiotherapy? J Clin Oncol 26:175-176, 2008

5. Fisher B, Anderson S, Bryant J, et al: Twenty-year follow-up of a randomized trial comparing total mastectomy, lumpectomy, and lumpectomy plus irradiation for the treatment of invasive breast cancer. N Engl J Med 347: 1233-1241, 2002

6. Farquhar C, Marjoribanks J, Lethaby A, et al: High-dose chemotherapy and autologous bone marrow or stem cell transplantation versus conventional chemotherapy for women with early poor prognosis breast cancer. Cochrane Database Syst Rev (5):CD003139, 2016

7. Wilson RR. Radiological use of fast protons. Radiology 47:487-491, 1946

8. Suit HD, Goitein M, Tepper J, et al: Exploratory study of proton radiation therapy using large field techniques and fractionated dose schedules. Cancer 35: 1646-1657, 1975

9. Lievens Y, Pijls-Johannesma M: Health economic controversy and costeffectiveness of proton therapy. Semin Radiat Oncol 23:134-141, 2013

10. Higgins KA, O'Connell K, Liu Y, et al: National Cancer Database analysis of proton versus photon radiation therapy in non-small cell lung cancer. Int $\mathrm{J}$ Radiat Oncol Biol Phys 97:128-137, 2017

11. Bradley JD, Paulus $R$, Komaki $R$, et al: Standard-dose versus high-dose conformal radiotherapy with concurrent and consolidation carboplatin plus paclitaxel with or without cetuximab for patients with stage IIIA or IIIB non-small-cell lung cancer (RTOG 0617): A randomised, two-by-two factorial phase 3 study. Lancet Oncol 16:187-199, 2015

12. Senan S, Brade A, Wang LH, et al: PROCLAIM: Randomized phase III trial of pemetrexed-cisplatin or etoposide-cisplatin plus thoracic radiation therapy followed by consolidation chemotherapy in locally advanced nonsquamous nonsmall-cell lung cancer. J Clin Oncol 34:953-962, 2016

13. Kong F-M, Ten Haken RK, Schipper $M$, et al: Effect of midtreatment PET/CTadapted radiation therapy with concurrent chemotherapy in patients with locally advanced non-small-cell lung cancer: A phase 2 clinical trial. JAMA Oncol 3: 1358-1365, 2017

14. Kong FM, Wang S. Nondosimetric risk factors for radiation-induced lung toxicity. Semin Radiat Oncol. 25:100-109, 2015

15. Palma DA, Senan S, Tsujino K, et al: Predicting radiation pneumonitis after chemoradiation therapy for lung cancer: An international individual patient data meta-analysis. Int J Radiat Oncol Biol Phys 85:444-450, 2013

16. Hanna N, Neubauer M, Yiannoutsos C, et al: Phase III study of cisplatin, etoposide, and concurrent chest radiation with or without consolidation docetaxel in patients with inoperable stage III non-small-cell lung cancer: The Hoosier Oncology Group and U.S. Oncology. J Clin Oncol 26: 5755-5760, 2008

17. Mailhot Vega R, Kim J, Hollander A, et al: Cost effectiveness of proton versus photon radiation therapy with respect to the risk of growth hormone deficiency in children. Cancer 121:1694-1702, 2015

18. Jin JY, Hu C, Xiao $Y$, et al: Higher radiation dose to immune system is correlated with poorer survival in patients with stage III non-small cell lung cancer: A secondary study of a phase 3 cooperative group trial (NRG Oncology RTOG 0617). Int J Radiat Oncol Biol Phys 99:S151S152, 2017

DOI: https://doi.org/10.1200/JCO.2017.76.5479; published at jco.org on January 12, 2018. 
AUTHOR'S DISCLOSURES OF POTENTIAL CONFLICTS OF INTEREST

What Happens When Proton Meets Randomization: Is There a Future for Proton Therapy?

The following represents disclosure information provided by authors of this manuscript. All relationships are considered compensated. Relationships are self-held unless noted. I = Immediate Family Member, Inst = My Institution. Relationships may not relate to the subject matter of this manuscript. For more information about ASCO's conflict of interest policy, please refer to www.asco.org/rwc or ascopubs.org/jco/site/ifc.

Feng-Ming (Spring) Kong

Honoraria: Varian Medical Systems

Speakers' Bureau: Varian Medical Systems

Research Funding: Varian Medical Systems

Travel, Accommodations, Expenses: Varian Medical Systems 
Editorial

\section{Acknowledgment}

I am grateful to Patrick Loehrer, MD, Theodore S. Lawrence, MD, PhD, Maria Werner-Wasik, MD, and Joel Tepper, MD, for their critical review and thoughtful comments. 